\title{
Exploring QCD Thermodynamics Using Möbius Fermions
}

\author{
Hantao Yin* \\ Department of Physics, Columbia University, New York, NY 10025, USA \\ E-mail: vinnhtephys.columbia.edu
}

We discuss the implementation of the HMC and RHMC evolution algorithms for $2+1$ flavors of Möbius fermions and present comparisons between Möbius fermions and DWF for QCD at zero and non-zero temperature. We discuss how quantities such as the residual mass, topological charge and chiral condensate behave using the Möbius action and compare the results with the standard domain wall fermions that we have previously used. The immediate benefit comes from a smaller size being used in the 5th dimension. With appropriately chosen Möbius parameters we argue that the same physics can be achieved more efficiently.

The 30 International Symposium on Lattice Field Theory - Lattice 2012,

June 24-29, 2012

Cairns, Australia

${ }^{*}$ Speaker. 


\section{Introduction}

The class of domain wall fermion actions maintains good chiral symmetry by making use of an extra 5th dimension. Increasing the size in this 5th dimension improves chiral behavior of the action, at the cost of demanding more computation resources since the total number of lattice sites is proportional to the size of the 5 th dimension.

The main motivation of using Möbius fermions is that it reduces the residual chiral symmetry breaking effect, while maintaining a much affordable size in the 5th dimension when compared to plain domain wall fermions.

\section{Measuring the Möbius Fermions Residual Mass}

We follow the convention used by [U, [1, []] when referring to the Möbius Dirac operator.

The residual chiral symmetry breaking effect of domain wall class fermion actions is usually measured by its residual mass, $m_{\text {res }}$. It is conventionally defined by evaluating the divergence of the axial current $A_{\mu}^{a}(x)$,

$$
\sum_{\mu} \Delta_{\mu} A_{\mu}^{a}(x)=2 m J_{5}^{a}(x)+2 J_{5 q}^{a}(x) .
$$

Where the $J_{5}^{a}(x)$ term measures the chiral symmetry breaking effect due to fermion mass, and $J_{5 q}^{a}(x)$ measures the residual chiral symmetry breaking effect due to the fermion action. When $2 J_{5 q}^{a}(x)$ is modeled by $J_{5}^{a}(x)$, the coefficient is recognized as $2 m_{\text {res }}$.

The actual form of $A_{\mu}^{a}(x), J_{5}^{a}(x)$ and $J_{5 q}^{a}(x)$ can be obtained by variating the path integral

$$
Z=\int \mathscr{D} U \mathscr{D} \bar{\psi} \mathscr{D} \psi \exp \left(-S_{G}(U)-\sum_{x s ; y s^{\prime}} \bar{\psi}_{x s} D_{x s ; y s^{\prime}} \psi_{y s^{\prime}}\right),
$$

where the detailed form of the Möbius Dirac operator $D_{x s ; y s^{\prime}}$ can be found in [四, Џ]. Following [ [由], we construct a local fermion variation

$$
\left\{\begin{array}{l}
\psi_{x s} \longleftarrow \exp \left(\varepsilon_{x s}^{a} \lambda^{a}\right) \psi_{x s} \\
\bar{\psi}_{x s} \longleftarrow \bar{\psi}_{x s} \exp \left(-\varepsilon_{x s}^{a} \lambda^{a}\right)
\end{array} .\right.
$$

The direct consequence of the above variation is the following conserved current, obtained from $\delta Z=0$,

$$
-\sum_{y s^{\prime}}\left\langle\bar{\psi}_{x s} \lambda^{a} D_{x s ; y s^{\prime}} \psi_{y s^{\prime}}\right\rangle+\sum_{y s^{\prime}}\left\langle\bar{\psi}_{y s^{\prime}} D_{y s^{\prime} ; x s} \lambda^{a} \psi_{x s}\right\rangle=0
$$

The above conserved current yields vector current $V_{\mu}(x)$ and axial current $A_{\mu}(x)$ that satisfy the following divergence relation,

$$
\begin{aligned}
\Delta_{\mu} V_{\mu}^{a}(x) & =0 \\
\Delta_{\mu} A_{\mu}^{a}(x) & =2 m J_{5}^{a}(x)+2 J_{5 q}^{a}(x) .
\end{aligned}
$$


The detailed form of $V_{\mu}^{a}(x)$ and $A_{\mu}^{a}(x)$ are complicated. But $J_{5}^{a}(x)$ and $J_{5 q}^{a}(x)$ are relatively simple ${ }^{12}$,

$$
\begin{aligned}
J_{5}^{a}(x) & =\sum_{y}\left(\bar{\psi}_{1}(y) \lambda^{a} D_{-}^{1}(y, x) P_{+} \psi_{2 N}(x)-\bar{\psi}_{2 N}(y) \lambda^{a} D_{-}^{2 N}(y, x) P_{-} \psi_{1}(x)\right) \\
J_{5 q}^{a}(x) & =\sum_{y}\left(\bar{\psi}_{N+1}(y) \lambda^{a} D_{-}^{N+1}(y, x) P_{+} \psi_{N}(x)-\bar{\psi}_{N}(y) \lambda^{a} D_{-}^{N}(y, x) P_{-} \psi_{N+1}(x)\right) .
\end{aligned}
$$

From the above $m_{\text {res }}$ can be readily computed using the ratio of $J_{5 q}^{a}(x)$ and $J_{5}^{a}(x)$.

We can move one step further and define the physical quark field for Möbius fermions as

$$
\begin{gathered}
q_{x}=P_{-} \psi_{x, 1}+P_{+} \psi_{x, 2 N} \\
\bar{q}_{x}=-\sum_{y} \bar{\psi}_{y, 2 N} D_{-}^{2 N}(y, x) P_{-}-\sum_{y} \bar{\psi}_{y, 1} D_{-}^{1}(y, x) P_{+} .
\end{gathered}
$$

Practically this implies replacing each occurance of $D$ (or $D^{-1}$ ) in a corresponding plain domain wall formula by $D_{-}^{-1} D$ (or $D^{-1} D_{-}$), where $D_{-}=\operatorname{diag}\left\{D_{-}^{1}, D_{-}^{2}, \cdots, D_{-}^{L_{s}}\right\}$. Another useful fact is that $\gamma_{5} R_{5} D_{-}^{-1} D$ is Hermitian for Möbius fermions if the parameters $b_{i}$ and $c_{i}$ are symmetric with respect to $i$, the 5th dimention index, i.e. $b_{i}=b_{L_{s}+1-i}$ and $c_{i}=c_{L_{s}+1-i}$.

Figure $\square$ shows $m_{\text {res }}$ of the Möbius fermion action measured on $16^{3} * 32, \beta=2.13, L_{s}=16$ DWF+I ensemble. We also set $L_{s}=16$ for the Möbius action, $b_{i}$ and $c_{i}$ are fixed to the same value for all $i$ and we further require that $b_{i}=c_{i}+1$. So there is only one free parameter, which is simply called $c$ in the graph. Effectively there is an optimal $c$ value when minimizing $m_{\text {res }}$ is concerned. This behavior is also confirmed by similar tests on other ensembles.

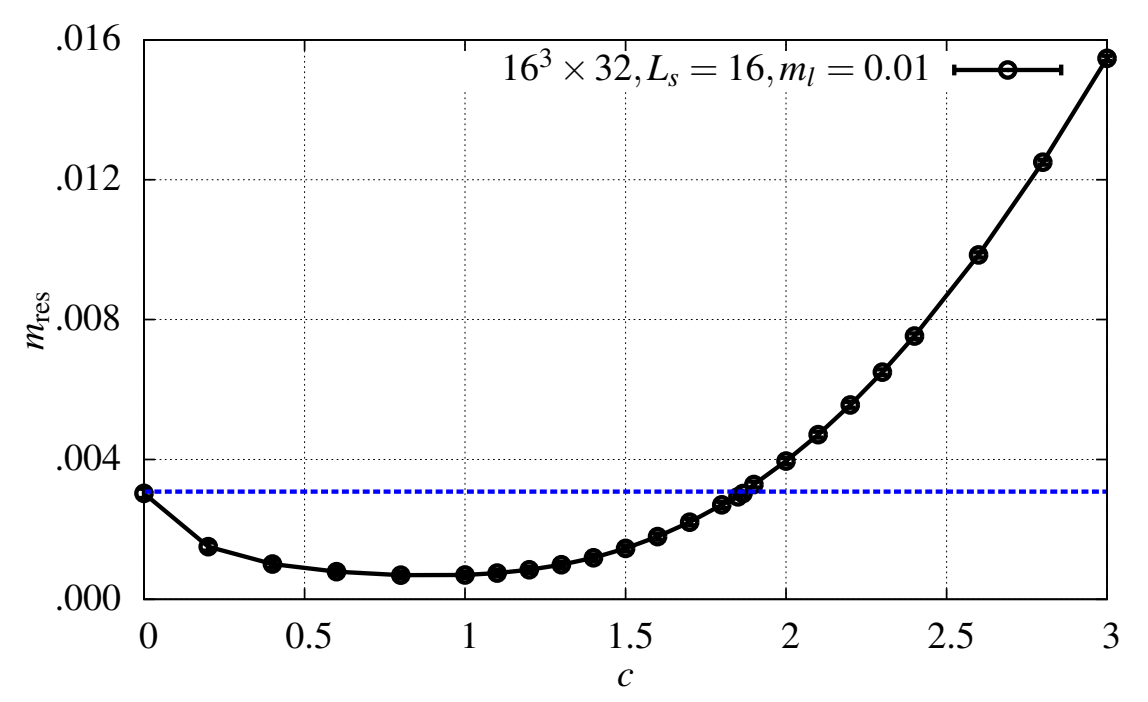

Figure 1: Residual Mass of the Möbius Dirac operator on plain domain wall fermion ensemble.

\subsection{Matching Two Möbius Actions}

In the following we consider only a simplified case of the Möbius fermions, where the parameters $b_{i}$ and $c_{i}$ are independent of the 5 th dimensional index $i$. And we simply denote the 2

\footnotetext{
${ }^{1}$ Both $L_{s}$ and $2 N$ refer to the size of the 5 th dimension of the Möbius fermions in this document.

${ }^{2}$ The definition of $D_{-}^{i}$ can be found in [四], where it is denoted by $D_{-}^{(i)}$.
} 
parameters as $b$ and $c$. The five dimensional Möbius Dirac operator can be reduced into a four dimensional equivalent overlap form. In this way we can compare two different Möbius Dirac operators.

Let the size of the 5 th dimension be $L_{s}$, we can apply the Domain wall-overlap transformation [四] and cast the Möbius Dirac operator into its four dimensional equivalent overlap form

$$
D_{O V}(m)=\frac{1}{2}\left(1+m+(1-m) \gamma_{5} \varepsilon(x)\right),
$$

where

$$
\begin{aligned}
\lambda & =b+c \\
x & =\gamma_{5} D_{W}\left(2+(b-c) D_{W}\right)^{-1},
\end{aligned}
$$

$D_{W}$ is the Wilson Dirac operator and $\varepsilon(x)$ is a rational approximation to the sign function

$$
\varepsilon(x)=\frac{(\lambda x+1)^{L_{s}}-(\lambda x-1)^{L_{s}}}{(\lambda x+1)^{L_{s}}+(\lambda x+1)^{L_{s}}} .
$$

Not surprisingly if two such approximations to the sign function $\varepsilon(x)$ are close to each other then their corresponding Möbius actions will share similar physical properties, this includes their $m_{\text {res }}$ and also other common quantities. In the following we show that such pairs of Möbius actions agree on measured quantities to very high accuracy.

To match two $\varepsilon(x)$ with different parameters $b, c, L_{s}$ and $b^{\prime}, c^{\prime}, L_{s}^{\prime}$, we only need to compare their Taylor expansions. This yields the following condition

$$
\begin{gathered}
L_{s}(b+c) \approx L_{s}^{\prime}\left(b^{\prime}+c^{\prime}\right) \\
b-c=b^{\prime}-c^{\prime} .
\end{gathered}
$$

The second condition ensures that the Shamir kernel $x$ is the same for the two actions. The above relation is useful for deriving Möbius parameters for actual ensemble generations.

Figure $\square$ shows the relative difference $\Delta \varepsilon(x)$ of 2 such $\varepsilon(x)$ functions. One rational approximation comes from $L_{s}=16$ plain domain wall fermion, and the other comes from $L_{s}=10$ Möbius fermion. The graph shows that if the parameters $b$ and $c$ are chosen according to ([2.14, 2.15) (the $\lambda=1.6$ case), then the induced Möbius fermion action agrees with the plain domain wall action within $10^{-3}$. 


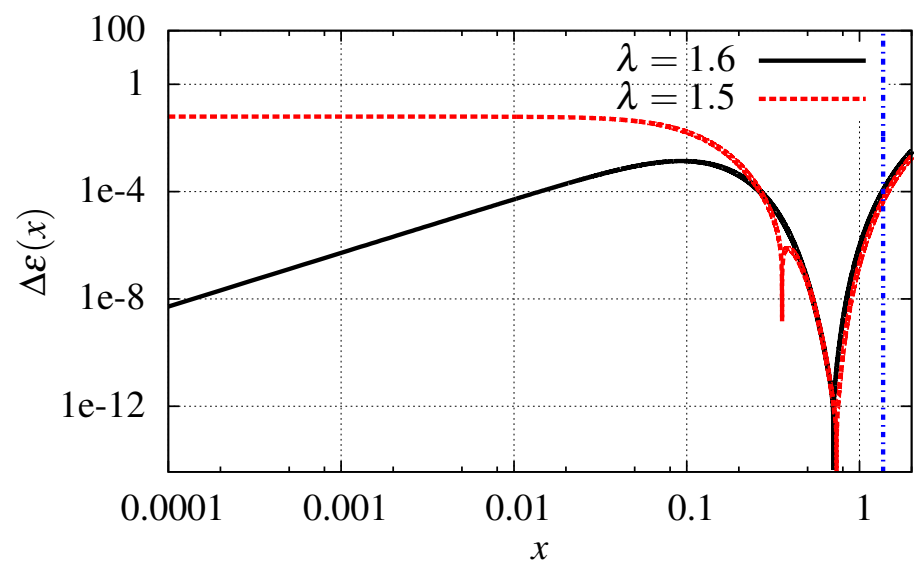

Figure 2: Comparison between $b+c=1, L_{s}=16$ Möbius operator (actually plain domain wall) and $b+c=$ $1.5,1.6, L_{s}=10$ Möbius operator.

\section{Möbius HMC Evolution}

We chose to generate a test $16^{3} * 8$ Möbius ensemble using $\beta=1.671$ with Iwasaki gauge action and the Dislocation Suppressing Determinant Ratio (DSDR). We choose the Möbius parameters to be $L_{s}=18, b=1.832$ and $c=0.832$ so it approximates plain domain wall fermion action with $L_{s}=48$ according to $(2.14,[2.55)$. We made this choice so we can directly compare this Möbius ensemble with one finite temperature lattice ensemble found in [可, where the corresponding $\beta=1.671, L_{s}=48$ ensemble was generated using plain domain wall fermion.

The summary of the 2 ensembles can be found in table $\mathbb{~}$. The 2 ensembles agree well on the plaquette values. The HMC algorithm runs on the Möbius ensemble approximately a factor of 2 faster, mainly due to the reduction in the size of the 5th dimension. Figure [ $]$ compares the evolution of topological charge of the 2 ensembles. It is clear that both ensembles show very similar properties.

\begin{tabular}{ccccc}
\hline ensemble & \# of trajectories & plaquette value & HMC time & HMC acceptance ratio \\
\hline$L_{s}=48 \mathrm{DWF}$ & 7000 & $0.484065(24)$ & $\sim 8000 \mathrm{sec}$ & 0.806 \\
$L_{s}^{\prime}=18$ Möbius & 1156 & $0.484048(50)$ & $\sim 4000 \mathrm{sec}$ & 0.849 \\
\hline
\end{tabular}

Table 1: Evolution summary of the $L_{s}=48$ plain domain wall ensemble and $L_{s}^{\prime}=18$ Möbius ensemble.

For the measurement of the chiral condensate $\langle\bar{\psi} \psi\rangle$, we measured on the Möbius ensemble using both the Möbius and plain domain wall fermion Dirac operators. For the Möbius fermions we use the quark field defined by $(2.8,2.9)$. The results from the two actions agree point by point, where the relative difference fluctuates around $0.1 \%$. 

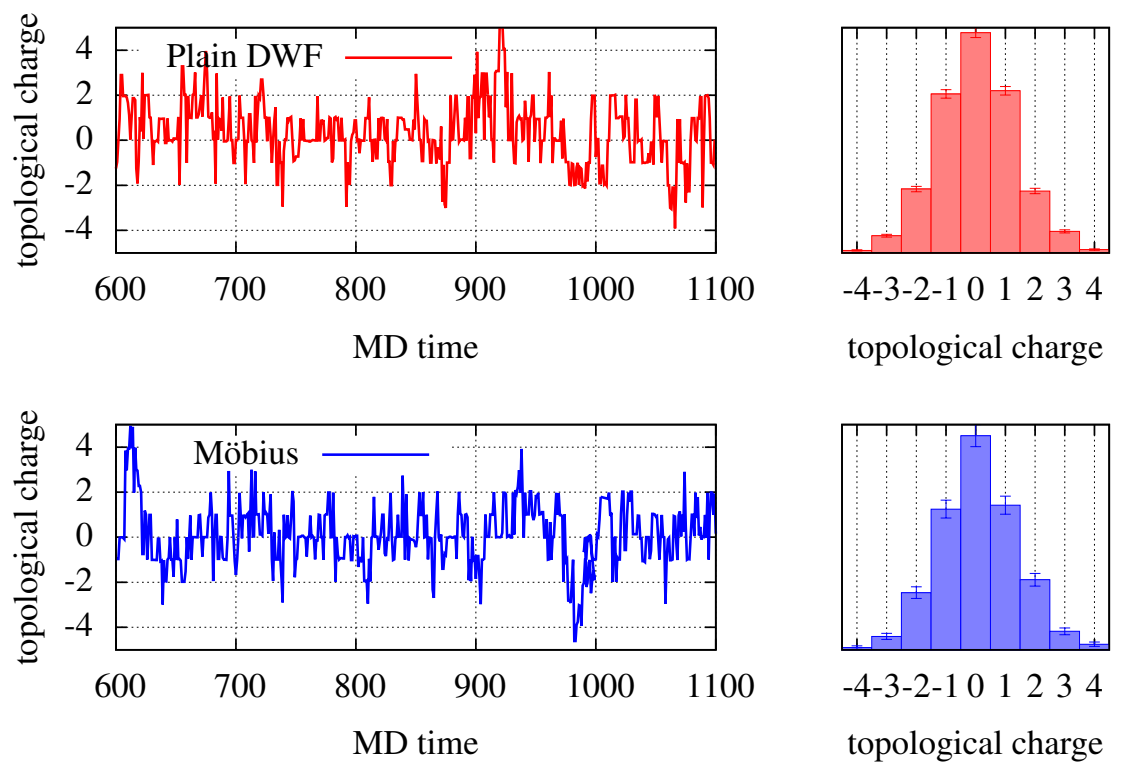

Figure 3: Comparison on topological charge evolution between $L_{s}=48$ plain domain wall ensemble and $L_{s}=18$ Möbius ensemble. Left: topological charge evolution history. Right: topological charge distribution.

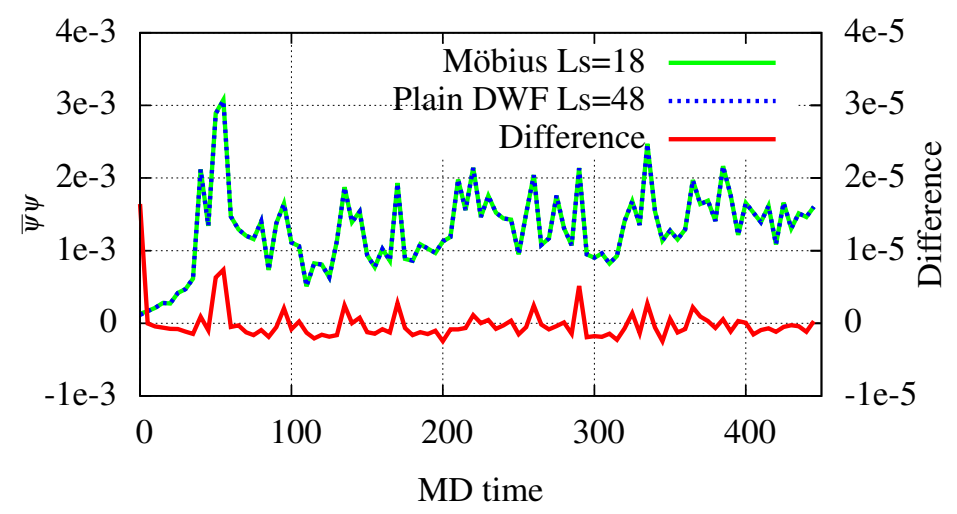

Figure 4: Comparison between the computed chiral condensate $\langle\bar{\psi} \psi\rangle$. Notice that there is a secondary scale for the difference.

\section{Möbius Fermions in QCD Thermodynamics}

The Möbius fermions are attractive for QCD Thermodynamics because it has good chiral symmetry property and is computationally cost effective compared to plain domain wall fermions or overlap fermions.

Table $\square$ shows the parameters used in the ongoing generation of $32^{3} \times 8$ Möbius fermion ensembles at physical pion mass by the HotQCD collaboration. The column $L_{s}(2 c+1)$ shows the size of $L_{s}$ necessary by plain domain wall fermions to achieve the same $m_{\mathrm{res}}$, which is a factor of $2 c+1$ larger compared to the Möbius fermions being used. 


\begin{tabular}{cc|ccccc}
\hline$\beta$ & $T(\mathrm{MeV})$ & $L_{s}$ & $c$ & $L_{s}(2 c+1)$ & $m_{l}\left(\times 10^{-5}\right)$ & $m_{\mathrm{res}}\left(\times 10^{-5}\right)$ \\
\hline 1.633 & 141 & 24 & 1.5 & 96.0 & 222 & $212(4)$ \\
1.671 & 150 & 16 & 1.5 & 64.0 & 206 & $169(3)$ \\
1.707 & 160 & 16 & 1.5 & 64.0 & 192 & $80(3)$ \\
1.740 & 169 & 16 & 1.2 & 54.4 & 179 & $53(3)$ \\
1.771 & 177 & 16 & 1.0 & 48.0 & 168 & $36(2)$ \\
1.801 & 187 & 16 & 1.0 & 48.0 & 158 & $25(2)$ \\
1.829 & 195 & 16 & 0.9 & 44.8 & 149 & $18(2)$ \\
\hline
\end{tabular}

Table 2: $m_{\text {res }}$ data for the ongoing $32^{3} \times 8$, physical pion mass QCD thermodynamics ensembles. The column $m_{l}$ is the target light quark mass and $m_{\text {res }}$ is the measured residual mass.

\section{Conclusion}

We presented a method to determine the residual chiral symmetry breaking effect, $m_{\text {res }}$ for Möbius fermions. We further presented simulation results of Möbius fermions on $16^{3} * 8$ finite temperature lattice and compared it with existing plain domain wall fermions. The results demonstrated that Möbius fermions can achieve similar physical properties with much smaller computational costs, mainly due to smaller sizes in its 5th dimension. We then showed how Möbius fermions reduce the cost of the ongoing generation of $32^{3} * 8$ finite temperature ensembles at physical pion mass.

\section{Acknowledgment}

We thank our colleagues in the RBC and HotQCD collaborations for discussions and tests on the program. We use the MDWF and BFM packages for simulations related to the Möbius fermions. This work is done using the New York Blue supercomputer at BNL and IBM Sequoia (BG/Q) at LLNL.

\section{References}

[1] R.C. Brower, H. Neff and K. Orginos, Möbius Fermions: Improved Domain Wall Chiral Fermions, Nucl. Phys. B(Proc. Suppl.) 140(2005) 686-688.

[2] R.C. Brower, H. Neff and K. Orginos, Möbius Fermions, Nucl. Phys. B(Proc. Suppl.) 153(2006) 191-198.

[3] R.C. Brower, H. Neff and K. Orginos, The Möbius Domain Wall Fermion Algorithm, arXiv: $1206.5214 \mathrm{v} 1$.

[4] V. Furman and Y. Shamir, Axial symmetries in lattice QCD with Kaplan fermions, Nucl.Phys. B439 (1995) 54-78.

[5] A. Bazavov et al., The chiral transition and $U(1)_{A}$ symmetry restoration from lattice $Q C D$ using Domain Wall Fermions, arXiv:1205.3535v1. 